\title{
Wigner function and pair production in parallel electric and magnetic fields
}

\author{
Xin-li Sheng, ${ }^{1,2}$ Ren-hong Fang, ${ }^{3}$ Qun Wang, ${ }^{1}$ and Dirk H. Rischke ${ }^{2,1}$ \\ ${ }^{1}$ Interdisciplinary Center for Theoretical Study and Department of Modern Physics, \\ University of Science and Technology of China, Hefei, Anhui 230026, China \\ ${ }^{2}$ Institute for Theoretical Physics, Goethe University, \\ Max-von-Laue-Str. 1, D-60438 Frankfurt am Main, Germany \\ ${ }^{3}$ Key Laboratory of Quark and Lepton Physics (MOE) and Institute of Particle Physics, \\ Central China Normal University, Wuhan, Hubei 430079, China
}

(Received 11 December 2018; published 8 March 2019)

\begin{abstract}
We derive analytical formulas for the equal-time Wigner function in an electromagnetic field of arbitrary strength. While the magnetic field is assumed to be constant, the electric field is assumed to be space independent and oriented parallel to the magnetic field. The Wigner function is first decomposed in terms of the so-called Dirac-Heisenberg-Wigner functions, and then the transverse-momentum dependence is separated using a new set of basis functions which depend on the quantum number $n$ of the Landau levels. Equations for the coefficients are derived and then solved for the case of a constant electric field. The pairproduction rate for each Landau level is calculated. In the case of finite temperature and chemical potential, the pair-production rate is suppressed by Pauli's exclusion principle.
\end{abstract}

DOI: 10.1103/PhysRevD.99.056004

\section{INTRODUCTION}

Quantum electrodynamics (QED) of strong electromagnetic (EM) fields has been studied for a very long time [1]. In the initial stage of noncentral heavy-ion collisions, the electromagnetic field can be as large as $9.8 \times 10^{22} \mathrm{~V} / \mathrm{m}$ at the Relativistic Heavy Ion Collider (RHIC) [2] and even larger at the LHC. Such extremely strong fields are generated by the fast-moving nuclei but will rapidly fall off with time [3]. The medium was estimated to extend the lifetime of the fields and enhance the possibility of detecting the influence of strong EM fields [4,5].

A strong magnetic field leads to interesting effects related to the chiral anomaly of quantum chromodynamics (QCD). On the other hand, a strong electric field can lead to decay of the QED vacuum. When the field strength is near or above the critical strength $E_{c}=m^{2} c^{3} / q \hbar$ [6-8], where $m$ is the mass of the particle and $q$ is its electric charge, particleantiparticle pairs can be created from vacuum. This process is commonly called the Schwinger process in honor of Julian Schwinger, who derived the pair-production rate in a famous work [8]. The rate is exponentially suppressed below the critical field strength, which is about $1.32 \times 10^{18} \mathrm{~V} / \mathrm{m}$ for electron-positron production. Pair production is a nonlinear

Published by the American Physical Society under the terms of the Creative Commons Attribution 4.0 International license. Further distribution of this work must maintain attribution to the author(s) and the published article's title, journal citation, and DOI. Funded by SCOAP. phenomenon, and the corresponding experiment is important for studying QED beyond the perturbative regime. The mechanism can occur in many systems such as in the early Universe, around neutron stars, and in heavy-ion collisions, while it is expected to appear in strong-laser experiments like the free-electron $\mathrm{x}$-ray laser $[9,10]$ and the extreme-light infrastructure (ELI) [11].

Although the Schwinger process has been studied for more than half a century, calculating the pair production in an arbitrary electromagnetic field is still a challenging problem. The case of a vanishing magnetic field $\mathbf{B}(t, \mathbf{x})=0$ and a space-independent electric field has been exhaustively discussed, and the problem can be translated into solving the famous Vlasov equation of quantum kinetic theory [12-14]. It can be analytically solved for a constant electric field $E(t)=E_{0}$ and the Sauter-type field $E(t)=$ $E_{0} \operatorname{sech}^{2}(t / \tau)$. Many theoretical methods are developed to deal with these two cases and go beyond these analytical benchmarks, such as directly through quantum field theory [8], Wentzel-Kramers-Brillouin methods [15-17], instanton methods [18-20], the Wigner-function method [21-23], the numerical worldline loop method [24,25], and holographic methods [26-28]. In principle, some methods such as the Wigner-function method [21-23] can be applied to very general cases, but one faces a system of nonlinear partial differential equations. However, the field configurations in cosmology or in heavy-ion collisions are much more complicated than the above-mentioned cases. One might find an approximate solution by partitioning spacetime into small cells and applying the analytical results for a 
constant electric field in each cell. This, however, may generate uncontrollable uncertainties because an instanton study [29] showed that temporal inhomogeneities tend to enhance the pair production while spatial ones tend to suppress it. Especially in heavy-ion collisions, where the EM fields vary rapidly in both space and time [5,30,31], a proper numerical treatment is necessary [32,33].

Nowadays, many researchers are focusing on the Schwinger process in strong-laser experiments [34,35]. The critical field strength $E_{c}$ for $e^{+} e^{-}$pair production corresponds to an average laser intensity $I_{c}=\frac{1}{2 \mu_{0} c} E_{c}^{2} \simeq$ $2.3 \times 10^{29} \mathrm{~W} / \mathrm{cm}^{2}$. Unfortunately, such a large intensity is difficult to generate in an experiment. In the ELI project [11], the laser pulse can only reach $\sim 10^{26} \mathrm{~W} / \mathrm{cm}^{2}$, which is three orders of magnitude lower than $I_{c}$. The pair production in such a case is strongly suppressed by a factor $\exp \left(-\pi E_{c} / E\right) \simeq 10^{-66}$. Clearly, the critical intensity $I_{c}$ is not attainable for laser experiments in the near future. Meanwhile, the electric field in heavy-ion collisions can reach $e E \sim m_{\pi}^{2} c^{3} / \hbar \gg e E_{c}$ at the RHIC [2], which provides realistic conditions to study pair production. In the recently discovered Dirac semimetals, massless Dirac fermions can be excited by an external electromagnetic field and may be experimentally observed through their transport properties. The production rate remains finite even if the Dirac fermions are massless [36], which is different from the Schwinger process in vacuum.

According to Maxwell's equations, a varying electric field will generate a magnetic field. Analytical calculations show that a magnetic field which is parallel to the electric field can increase the pair-production rate [16,37-40]. Recently, the enhancement of the pair-production rate due to parallel magnetic fields has been studied in string theory [41-43]. The pair-production rate is modified by the thermal medium [44-46]. In this paper, we will reproduce these results via the Wigner-function method. On the other hand, the pair production in parallel electromagnetic fields is related to the chiral anomaly $[47,48]$ and to pseudoscalar condensation [49-51], which can be verified using the results of this paper. We will focus on these effects in future work.

This paper is organized as follows. In Sec. II, we will briefly introduce the equal-time Wigner function and the Dirac-Heisenberg-Wigner (DHW) functions. General equations of motion for the DHW functions are also listed in this section. In Sec. III, we simplify the equations of motion for the DHW functions in a spatially homogeneous electric field and give analytical solutions for a constant electric field. A constant magnetic field, which is parallel to the electric field, is taken into account in Sec. IV. The DHW functions reflect the behavior of the Landau levels. Analytical solutions are derived when both electric and magnetic fields are constant. In Sec. V, we read off the pairproduction rate from the DHW functions derived in Sec. IV. In Sec. VI, we give a summary and provide an outlook for future work. Details about the auxiliary functions used in this paper and their properties are summarized in Appendix.

We take fermions to have positive unit charge $q=+e$ and the electric and magnetic fields to point in the $z$-direction. We use the following notations for four-vectors: $X=\left(x^{\mu}\right)=(t, \mathbf{r})=\left(t, \mathbf{x}_{T}, z\right)=(t, x, y, z)$ and $P=\left(p^{\mu}\right)=$ $(E, \mathbf{p})=\left(E, \mathbf{p}_{T}, p_{z}\right)=\left(E, p_{x}, p_{y}, p_{z}\right)$. We also use the differential operators $\partial_{t}=\frac{\partial}{\partial t}, \nabla_{\mathbf{x}}=\left(\frac{\partial}{\partial x}, \frac{\partial}{\partial z}, \frac{\partial}{\partial z}\right)$ and $\boldsymbol{\nabla}_{\mathbf{p}}=$ $\left(\partial_{p_{x}}, \partial_{p_{y}}, \partial_{p_{z}}\right)=\left(\frac{\partial}{\partial p_{x}}, \frac{\partial}{\partial p_{y}}, \frac{\partial}{\partial p_{z}}\right)$. Our units are natural Heaviside-Lorentz units, $\hbar=c=k_{B}=\epsilon_{0}=\mu_{0}=1$. The metric tensor is $g_{\mu \nu}=\operatorname{diag}(+,-,-,-)$.

\section{DHW FUNCTIONS AND THEIR EQUATIONS OF MOTION}

In this section, we define the DHW functions as expansion coefficients of the equal-time Wigner function. The choice of the gauge potential is to some degree arbitrary. Here, we use the temporal gauge $A^{0}=0$, for which the EM fields are given by $\mathbf{E}=-\partial_{t} \mathbf{A}$ and $\mathbf{B}=\boldsymbol{\nabla} \times \mathbf{A}$. In principle, the EM fields include contributions from external fields and contributions from all charged particles. But in this paper, we will focus on the case of an external field only and neglect the interaction between particles, which corresponds to a free Fermi gas.

The gauge-invariant Wigner operator is given by

$$
\begin{aligned}
\hat{W}(X, P)= & \int \frac{d^{4} Y}{(2 \pi)^{4}} \exp \left(-i y^{\mu} p_{\mu}\right) \bar{\psi}\left(X+\frac{Y}{2}\right) \\
& \otimes U\left(X+\frac{Y}{2}, X-\frac{Y}{2}\right) \psi\left(X-\frac{Y}{2}\right),
\end{aligned}
$$

where $\psi$ is the Dirac field operator for spin-1/2 particles. This formula represents the Fourier transform with respect to the relative position $Y$ of the direct product of two fermion field operators at space-time points $X+\frac{Y}{2}$ and $X-\frac{Y}{2}$, respectively. The gauge link between these two points renders the Wigner operator gauge invariant and is defined as

$U\left(X+\frac{Y}{2}, X-\frac{Y}{2}\right)=\exp \left[-i e y^{\mu} \int_{-1 / 2}^{1 / 2} d s A_{\mu}(X+s Y)\right]$,

where $A_{\mu}$ is the gauge potential, e.g., in this paper the electromagnetic potential. Taking the expectation value of the Wigner operator in a state $|\Omega\rangle$, we obtain the Wigner function

$$
W(X, P) \equiv\langle\Omega|\hat{W}(X, P)| \Omega\rangle .
$$

The Wigner function, defined in eight-dimensional phase space $\left(x^{\mu}, p^{\mu}\right)$, is Lorentz covariant but does not have a 
clear physical interpretation [52,53]. By integrating over the energy $p^{0}$, we obtain the corresponding equal-time Wigner function [54-56], which can be interpreted as a quasiprobability distribution in six-dimensional phase space $(\mathbf{x}, \mathbf{p})$ at time $t$. Such a procedure evidently breaks the Lorentz covariance; however, the equation of motion might simplify to that of an initial-value problem. On the other hand, we adopt the Hartree approximation; i.e., we treat the quantum EM field as a semiclassical EM field, from which we derive the following formula for the equaltime Wigner function $W(t, \mathbf{x}, \mathbf{p})$ :

$$
\begin{aligned}
& W(t, \mathbf{x}, \mathbf{p}) \\
& =\int \frac{d^{3} \mathbf{y}}{(2 \pi)^{3}} \exp \left[i \mathbf{y} \cdot \mathbf{p}+i e \int_{-1 / 2}^{1 / 2} d s \mathbf{y} \cdot \mathbf{A}(t, \mathbf{x}+s \mathbf{y})\right] \\
& \quad \times\left\langle\Omega\left|\bar{\psi}\left(t, \mathbf{x}+\frac{\mathbf{y}}{2}\right) \otimes \psi\left(t, \mathbf{x}-\frac{\mathbf{y}}{2}\right)\right| \Omega\right\rangle .
\end{aligned}
$$

The Hartree approximation ignores higher-loop radiative corrections and is a good approximation for strong EM fields.

The equation of motion for the equal-time Wigner function can be derived from the Dirac equation. We consider a nonzero chemical potential $\mu$ associated with the conservation of fermion number, which, for the sake of simplicity, we assume to be constant in space-time; hence, its derivatives vanish. An effective way to include the chemical potential is by adding a term $+\mu \hat{N}$ to the DiracHamilton operator $\hat{H}_{D}$, where $\hat{N}$ is the fermion-number operator. The corresponding Dirac equation reads

$$
\left[i \gamma^{\sigma}\left(\partial_{\sigma}+i e A_{\sigma}\right)-m+\mu \gamma^{0}\right] \psi(X)=0 .
$$

Taking the time derivative of Eq. (4) and simplifying the result using the Dirac equation, we obtain the following equation of motion [21],

$D_{t} W=\frac{1}{2} \mathbf{D}_{\mathbf{x}} \cdot\left[W, \boldsymbol{\gamma}^{0} \boldsymbol{\gamma}\right]-i \boldsymbol{\Pi} \cdot\left\{W, \gamma^{0} \boldsymbol{\gamma}\right\}+i m\left[W, \gamma^{0}\right]$,

where the operators $D_{t}, \mathbf{D}_{\mathbf{x}}$, and $\boldsymbol{\Pi}$ are generalized operators for time and spatial derivatives, as well as momentum, in the presence of an EM field,

$$
\begin{aligned}
D_{t} & \equiv \partial_{t}+e \int_{-1 / 2}^{1 / 2} d s \mathbf{E}\left(t, \mathbf{x}-i s \boldsymbol{\nabla}_{\mathbf{p}}\right) \cdot \boldsymbol{\nabla}_{\mathbf{p}} \\
\mathbf{D}_{\mathbf{x}} & \equiv \boldsymbol{\nabla}_{\mathbf{x}}+e \int_{-1 / 2}^{1 / 2} d s \mathbf{B}\left(t, \mathbf{x}-i s \boldsymbol{\nabla}_{\mathbf{p}}\right) \times \boldsymbol{\nabla}_{\mathbf{p}} \\
\boldsymbol{\Pi} & \equiv \mathbf{p}+i e \int_{-1 / 2}^{1 / 2} d s s \mathbf{B}\left(t, \mathbf{x}-i s \boldsymbol{\nabla}_{\mathbf{p}}\right) \times \boldsymbol{\nabla}_{\mathbf{p}} .
\end{aligned}
$$

For spatially homogeneous EM fields $F^{\mu \nu}(t, \mathbf{x})=F^{\mu \nu}(t)$, these operators become local,

$$
\begin{aligned}
D_{t} & =\partial_{t}+e \mathbf{E}(t) \cdot \nabla_{\mathbf{p}} \\
\mathbf{D}_{\mathbf{x}} & =\boldsymbol{\nabla}_{\mathbf{x}}+e \mathbf{B}(t) \times \boldsymbol{\nabla}_{\mathbf{p}}, \\
\boldsymbol{\Pi} & =\mathbf{p}
\end{aligned}
$$

It can be easily checked that the equal-time Wigner function $W(t, \mathbf{x}, \mathbf{p})$ satisfies $W^{\dagger}=\gamma^{0} W \gamma^{0}$ and can be decomposed in terms of the 16 independent generators of the Clifford algebra $\Gamma_{i}=\left\{1, i \gamma^{5}, \gamma^{\mu}, \gamma^{5} \gamma^{\mu}, \frac{1}{2} \sigma^{\mu \nu}\right\}$,

$$
W(t, \mathbf{x}, \mathbf{p})=\frac{1}{4}\left(\mathcal{F}+i \gamma^{5} \mathcal{P}+\gamma^{\mu} \mathcal{V}_{\mu}+\gamma^{5} \gamma^{\mu} \mathcal{A}_{\mu}+\frac{1}{2} \sigma^{\mu \nu} \mathcal{S}_{\mu \nu}\right)
$$

where $\sigma^{\mu \nu}=\frac{i}{2}\left[\gamma^{\mu}, \gamma^{\nu}\right]$ is the antisymmetric spin tensor. These 16 functions, commonly called DHW functions, are real functions of time $t$ and six-dimensional phase space $(\mathbf{x}, \mathbf{p})$. The tensor part can be further decomposed into two vector functions

$$
\boldsymbol{T}=\left(\begin{array}{c}
\mathcal{S}^{10} \\
\mathcal{S}^{20} \\
\mathcal{S}^{30}
\end{array}\right), \quad \boldsymbol{S}=\left(\begin{array}{c}
\mathcal{S}^{23} \\
\mathcal{S}^{31} \\
\mathcal{S}^{12}
\end{array}\right)
$$

Some of these DHW functions have a clear physical meaning [54]; e.g., $\mathcal{F}$ determines the mass density, $\mathcal{V}_{\mu}$ is the vector-charge current density, $\mathcal{A}_{\mu}$ is the chiral-charge current density, and $S$ is the magnetic-moment density. Substituting Eq. (9) into the equation of motion (6) and projecting onto the 16 basis matrices, we find a system of partial differential equations (PDEs) for the DHW functions

$$
D_{t}\left(\begin{array}{l}
\boldsymbol{G}_{1} \\
\boldsymbol{G}_{2} \\
\boldsymbol{G}_{3} \\
\boldsymbol{G}_{4}
\end{array}\right)=\left(\begin{array}{cccc}
0 & 0 & 0 & M_{1} \\
0 & 0 & -M_{2} & 0 \\
0 & -M_{2} & 0 & -2 m \\
-M_{1} & 0 & 2 m & 0
\end{array}\right)\left(\begin{array}{l}
\boldsymbol{G}_{1} \\
\boldsymbol{G}_{2} \\
\boldsymbol{G}_{3} \\
\boldsymbol{G}_{4}
\end{array}\right),
$$

where the DHW functions have been divided into four groups and each group is composed of four functions [57],

$$
\begin{aligned}
\boldsymbol{G}_{1}=\left(\begin{array}{c}
\mathcal{F} \\
\boldsymbol{S}
\end{array}\right), & \boldsymbol{G}_{2}=\left(\begin{array}{c}
\mathcal{V}_{0} \\
\mathcal{A}
\end{array}\right), \\
\boldsymbol{G}_{3}=\left(\begin{array}{c}
\mathcal{A}_{0} \\
\mathcal{V}
\end{array}\right), & \boldsymbol{G}_{4}=\left(\begin{array}{c}
\mathcal{P} \\
\boldsymbol{T}
\end{array}\right) .
\end{aligned}
$$

In Eq. (11), we have introduced the two matrices

$$
M_{1} \equiv\left(\begin{array}{cc}
0 & 2 \boldsymbol{\Pi}^{T} \\
2 \boldsymbol{\Pi} & \mathbf{D}_{\mathbf{x}}^{\times}
\end{array}\right), \quad M_{2} \equiv\left(\begin{array}{cc}
0 & \mathbf{D}_{\mathbf{x}}^{T} \\
\mathbf{D}_{\mathbf{x}} & -2 \boldsymbol{\Pi}^{\times}
\end{array}\right),
$$

where $\boldsymbol{\Pi}$ and $\mathbf{D}_{\mathbf{x}}$ were already defined in Eq. (7). For any three-dimensional column vector $\mathbf{V}, \mathbf{V}^{T}$ is the 
corresponding transposed vector (line vector), and $\mathbf{V}^{\times}$ represents the antisymmetric $3 \times 3$ matrix

$$
\mathbf{V}^{\times}=\left(\begin{array}{ccc}
0 & -V_{z} & V_{y} \\
V_{z} & 0 & -V_{x} \\
-V_{y} & V_{x} & 0
\end{array}\right),
$$

the elements of which are $\mathbf{V}_{i j}^{\times}=-\epsilon_{i j k} V_{k}$. The differential equations (11) are equivalent to the ones in Refs. [21,55], but here we write them in a matrix form. When dealing with the Landau levels in a constant magnetic field, this matrix form allows for more compact formulas [57].

\section{SPATIALLY HOMOGENEOUS ELECTRIC FIELD}

In this section, we will simplify the equations of motion (11) for the DHW functions in a spatially homogeneous electric field and then give the solution for a constant electric field. The electric field is taken to point into the $z$-direction. In this case, the gauge potential is $\mathbf{A}(t)=$ $A(t) \mathbf{e}_{z}$ with $\partial_{t} A(t)=-E(t)$. A similar procedure has been adopted in Ref. [21], in which the authors only discussed the pair production in vacuum. In a thermal environment, the low-energy states are occupied, which blocks the production of pairs into these states. In this section, a thermal equilibrium distribution is assumed at the initial time. Since collisions between particles are not included, all existing particles are accelerated by the electric field, and thus the distribution depends on the canonical momentum. We show that, in the solution, the thermal distribution appears as an overall suppression factor, which does not influence the structure of the PDE system. The basis used in this section is different from the one in Ref. [21], but both span the same Hilbert space and thus are equivalent to each other. The system of PDEs and corresponding initial conditions derived with the basis in this section provides a convenient framework to describe pair production in parallel electric and magnetic fields in Sec. IV.

Let us first consider the DHW functions for a free gas of fermions. These can be derived by first quantizing the field operators in terms of solutions for free particles, which can be found in any textbook of quantum field theory, and then inserting the field operators into the definition of the Wigner function. The result is

$$
\begin{aligned}
\left(\begin{array}{c}
\mathcal{F} \\
\mathcal{V}
\end{array}\right)_{\text {free }}(\mathbf{p})= & \frac{d_{s}}{(2 \pi)^{3}} \frac{1}{E_{\mathbf{p}}}\left[f_{\mathrm{FD}}\left(E_{\mathbf{p}}-\mu\right)+f_{\mathrm{FD}}\left(E_{\mathbf{p}}+\mu\right)-1\right] \\
& \times\left(\begin{array}{c}
m \\
\mathbf{p}
\end{array}\right), \\
\mathcal{V}_{0, \text { free }}(\mathbf{p})= & \frac{d_{s}}{(2 \pi)^{3}}\left[f_{\mathrm{FD}}\left(E_{\mathbf{p}}-\mu\right)-f_{\mathrm{FD}}\left(E_{\mathbf{p}}+\mu\right)+1\right] .
\end{aligned}
$$

Here, $d_{s}$ is the degeneracy of spin, which is $d_{s}=2$ for spin- $\frac{1}{2}$ particles, and

$$
f_{\mathrm{FD}}\left(E_{\mathbf{p}} \mp \mu\right)=\frac{1}{1+\exp \left[\beta\left(E_{\mathbf{p}} \mp \mu\right)\right]}
$$

is the Fermi-Dirac distribution for particles/antiparticles with energy $E_{\mathbf{p}}$ and vector chemical potential $\mu$, while $\beta=T^{-1}$ is the inverse temperature. Note that the fermionic field operators in the definition (4) are not normal ordered; therefore, taking the expectation value in the state $|\Omega\rangle$ yields an additional $\mp 1$, which appears in the square brackets in Eq. (15). Here, $\mathcal{V}_{0}, \mathcal{V}$, and $\mathcal{F}$ are the charge, current, and mass densities, respectively. All other DHW functions vanish for a free gas of fermions, $\mathcal{P}=\mathcal{A}_{0}=$ $\mathcal{A}=\mathbf{S}=\mathbf{T}=0$, which can be proven using the completeness relations for the Dirac spinors $u(\mathbf{k}, s)$ and $v(\mathbf{k}, s)$.

We now proceed to solve the equations of motion (11) for the DHW functions. Due to the absence of a magnetic field and translation invariance of the system, we can set the spatial derivative $\mathbf{D}_{\mathbf{x}}$ to zero, and $\boldsymbol{\Pi} \equiv \mathbf{p}$. The matrices in Eq. (13) then simplify to

$M_{1}=\left(\begin{array}{cc}0 & 2 \mathbf{p}^{T} \\ 2 \mathbf{p} & \mathbf{0}_{3 \times 3}\end{array}\right), \quad M_{2}=\left(\begin{array}{cc}0 & \mathbf{0}_{1 \times 3} \\ \mathbf{0}_{3 \times 1} & -2 \mathbf{p}^{\times}\end{array}\right)$,

and $D_{t}=\partial_{t}+e E(t) \partial_{p_{z}}$. Then, the 16 equations of motion for the DHW functions can be divided into several groups. The equation for the charge density separates from the others and reads

$$
D_{t} \mathcal{V}_{0}(t, \mathbf{p})=0
$$

After integrating over the momentum $\mathbf{p}$ and neglecting the boundary terms (because there is no particle with infinite $p_{z}$ ), the above equation is nothing but the conservation of net charge. Furthermore, the ten equations of motion for the DHW functions $\mathcal{F}, \mathcal{V}, \mathcal{A}$, and $\boldsymbol{T}$ decouple from the other five for the functions $\mathcal{P}, \mathcal{A}_{0}$, and $\mathbf{S}$. These latter ones will no longer be considered because their initial values are zero and thus they will remain zero for later times as well. In matrix form, we have

$$
D_{t} \boldsymbol{w}(t, \mathbf{p})=M(\mathbf{p}) \boldsymbol{w}(t, \mathbf{p}),
$$

where $\boldsymbol{w}(t, \mathbf{p})=(\mathcal{F}, \mathcal{V}, \mathcal{A}, \boldsymbol{T})^{T}$ is a ten-dimensional vector consisting of ten DHW functions and $M(\mathbf{p})$ is a $10 \times 10$ matrix,

$$
M(\mathbf{p})=2\left(\begin{array}{cccc}
0 & 0 & 0 & \mathbf{p}^{T} \\
0 & 0 & \mathbf{p}^{\times} & -m \\
0 & \mathbf{p}^{\times} & 0 & 0 \\
-\mathbf{p} & m & 0 & 0
\end{array}\right) .
$$


Inspired by the form (15) of the free DHW functions, we make the following ansatz for the solution of Eq. (19):

$$
\begin{aligned}
\boldsymbol{w}(t, \mathbf{p})= & \frac{d_{s}}{(2 \pi)^{3}}\left\{f_{\mathrm{FD}}\left(E_{\mathbf{p}+e \delta A(t) \mathbf{e}_{z}}-\mu\right)\right. \\
& \left.+f_{\mathrm{FD}}\left(E_{\mathbf{p}+e \delta A(t) \mathbf{e}_{z}}+\mu\right)-1\right\} \sum_{i=1}^{3} \chi_{i}(t, \mathbf{p}) \mathbf{e}_{i}\left(\mathbf{p}_{T}\right) .
\end{aligned}
$$

Here, $\delta A(t) \equiv A(t)-A\left(t_{0}\right)$ is the difference of the gauge potentials at time $t$ and at initial time $t_{0}$. The distribution thus depends on the canonical momentum, which reflects the acceleration of fermions in an electric field. Since the operator $D_{t}$ acting on $\mathbf{p}+e \delta A(t) \mathbf{e}_{z}$ gives zero, the term in the curly brackets in Eq. (21) behaves like a constant overall factor and can be taken out of Eq. (19). The value of this term is in the range $(-1,0)$, which is the effect of Pauli blocking by particles already present in the thermal system. Note that, since the matrix $\boldsymbol{w}(t, \mathbf{p})$ has dimension 10 , in principle, we would need ten basis vectors $\mathbf{e}_{i}$ in the ansatz (21). However, we actually only need three because these form a closed subspace under the operators $D_{t}$ and $M(\mathbf{p})$, while the initial conditions are also inside this subspace. These basis vectors are

$$
\begin{aligned}
\boldsymbol{e}_{1}= & \left(\begin{array}{c}
0 \\
\mathbf{e}_{z} \\
\mathbf{0} \\
\mathbf{0}
\end{array}\right), \quad \boldsymbol{e}_{2}\left(\mathbf{p}_{T}\right)=\frac{1}{m_{T}}\left(\begin{array}{c}
m \\
\mathbf{p}_{T} \\
\mathbf{0} \\
\mathbf{0}
\end{array}\right), \\
\boldsymbol{e}_{3}\left(\mathbf{p}_{T}\right) & =\frac{1}{m_{T}}\left(\begin{array}{c}
0 \\
\mathbf{0} \\
\mathbf{e}_{z} \times \mathbf{p}_{T} \\
-m \mathbf{e}_{z}
\end{array}\right),
\end{aligned}
$$

which are independent of $t$ and $p_{z}$, so that $D_{t} \boldsymbol{e}_{i}=0$ for all $i=1,2,3$. Here, we have introduced the transverse mass $m_{T} \equiv \sqrt{m^{2}+\mathbf{p}_{T}^{2}}$, so that the three basis vectors are properly normalized, $\boldsymbol{e}_{i} \cdot \boldsymbol{e}_{j}=\delta_{i j}$. We can also check that they are closed under the operator $M(\mathbf{p})$,

$$
M(\mathbf{p})\left(\begin{array}{l}
\boldsymbol{e}_{1} \\
\boldsymbol{e}_{2} \\
\boldsymbol{e}_{3}
\end{array}\right)=2\left(\begin{array}{ccc}
0 & 0 & -m_{T} \\
0 & 0 & p_{z} \\
m_{T} & -p_{z} & 0
\end{array}\right)\left(\begin{array}{l}
\boldsymbol{e}_{1} \\
\boldsymbol{e}_{2} \\
\boldsymbol{e}_{3}
\end{array}\right) .
$$

Inserting the ansatz (21) into Eq. (19) and using Eq. (23), we can derive the equations of motion for the coefficient functions $\chi_{i}(t)$,

$$
D_{t}\left(\begin{array}{l}
\chi_{1} \\
\chi_{2} \\
\chi_{3}
\end{array}\right)(t, \mathbf{p})=2\left(\begin{array}{ccc}
0 & 0 & m_{T} \\
0 & 0 & -p_{z} \\
-m_{T} & p_{z} & 0
\end{array}\right)\left(\begin{array}{l}
\chi_{1} \\
\chi_{2} \\
\chi_{3}
\end{array}\right)(t, \mathbf{p}) \text {. }
$$

In order to solve this system of PDEs, we need to specify the initial condition. Here, we choose the values of the DHW functions in the absence of an electric field. For an integrable electric field, which vanishes sufficiently rapidly for $t \rightarrow \pm \infty$, such as the Sauter-type field $E(t)=E_{0} \cosh ^{-2}(t / \tau)$, we specify the initial condition for $t_{0} \rightarrow-\infty$, where we take the DHW functions to assume the values given by Eq. (15).

However, for a constant electric field $E(t)=E_{0}$, the momentum shift will be infinitely large if we take $t_{0} \rightarrow-\infty$, because a constant field is not integrable. In reality, fermions will collide with each other, kinetic energy will be converted to thermal energy, and the system will approach thermodynamical equilibrium. Here, we make the assumption that the system is already in thermodynamical equilibrium at initial time $t_{0}$. We should find a solution that coincides with Eq. (15) when the field strength is sufficiently small, $E_{0} \rightarrow 0$, i.e.,

$$
\left.\left(\begin{array}{c}
\chi_{1} \\
\chi_{2} \\
\chi_{3}
\end{array}\right)(t, \mathbf{p})\right|_{E_{0} \rightarrow 0}=\frac{1}{E_{\mathbf{p}}}\left(\begin{array}{c}
p_{z} \\
m_{T} \\
0
\end{array}\right) .
$$

The pair-production rate and the corresponding Wigner function have analytical solutions for both a constant field and a Sauter-type field; see Ref. [21] for details of the derivation from quantum kinetic theory, which we will not repeat here. In a constant field, $E(t)=E_{0}$, the solutions do not depend on space-time coordinates, which is obvious because of translation invariance,

$$
\left(\begin{array}{l}
\chi_{1} \\
\chi_{2} \\
\chi_{3}
\end{array}\right)(\mathbf{p})=\left(\begin{array}{c}
d_{1}\left(\eta, \sqrt{\frac{2}{e E_{0}}} p_{z}\right) \\
\frac{m_{T}}{\sqrt{2 e E_{0}}} d_{2}\left(\eta, \sqrt{\frac{2}{e E_{0}}} p_{z}\right) \\
\frac{m_{T}}{\sqrt{2 e E_{0}}} d_{3}\left(\eta, \sqrt{\frac{2}{e E_{0}}} p_{z}\right)
\end{array}\right)
$$

where $\eta \equiv m_{T}^{2} /\left(e E_{0}\right)$ is the dimensionless transverse mass square and the auxiliary functions are listed in Eq. (A1) of Appendix. It is easy to check numerically that the solutions (26) satisfy the constraint (25) and the system (24) of PDEs. The corresponding DHW functions can be readily derived by inserting Eq. (26) into Eq. (21). 


\section{PARALLEL AND SPATIALLY HOMOGENEOUS ELECTRIC AND MAGNETIC FIELDS}

In this section, we will consider spatially homogeneous electric and magnetic fields which are parallel to each other. Without loss of generality, the fields are assumed to point into the $z$-direction. We also assume the magnetic field to be constant in time. Then, the solution can be simplified by separately considering the different Landau levels. We provide an analytical solution for the case when the electric field is also constant in time.

\section{A. Initial conditions}

Analogously to the case without magnetic field, we choose the DHW functions in a pure magnetic field as an initial condition for the system (11) of PDEs. Since we consider this field to be constant in space and time, an analytical solution can be found. The corresponding covariant DHW functions in eight-dimensional phase space have been determined in Ref. [57]. In this paper, we set the axial chemical potential to zero; i.e., we do not consider the chiral magnetic effect. Then, using the results of Ref. [57], the covariant DHW functions read

$$
\begin{aligned}
\left(\begin{array}{c}
\boldsymbol{G}_{1}(P) \\
\boldsymbol{G}_{2}(P)
\end{array}\right)= & \sum_{n=0} V^{(n)}\left(p_{0}, p_{z}\right) \boldsymbol{e}_{1}^{(n)}\left(p_{T}\right)\left(\begin{array}{c}
m \\
p_{0}+\mu
\end{array}\right), \\
\boldsymbol{G}_{3}(P)= & p_{z} V^{(0)}\left(p_{0}, p_{z}\right) \mathbf{e}_{1}^{(0)}\left(p_{T}\right) \\
& +\sum_{n>0} V^{(n)}\left(p_{0}, p_{z}\right)\left[p_{z} \boldsymbol{e}_{2}^{(n)}\left(p_{T}\right)\right. \\
& \left.+\sqrt{2 n e B} \boldsymbol{e}_{3}^{(n)}\left(\mathbf{p}_{T}\right)\right], \\
\boldsymbol{G}_{4}(P)= & 0,
\end{aligned}
$$

where

$$
\begin{aligned}
V^{(n)}\left(p_{0}, p_{z}\right)= & \frac{2\left(2-\delta_{n 0}\right)}{(2 \pi)^{3}} \delta\left\{\left(p_{0}+\mu\right)^{2}-\left[E_{p_{z}}^{(n)}\right]^{2}\right\} \\
& \times\left\{\theta\left(p_{0}+\mu\right) f_{\mathrm{FD}}\left(p_{0}\right)\right. \\
& \left.+\theta\left(-p_{0}-\mu\right)\left[f_{\mathrm{FD}}\left(-p_{0}\right)-1\right]\right\} .
\end{aligned}
$$

Here, $E_{p_{z}}^{(n)}=\sqrt{m^{2}+p_{z}^{2}+2 n e B}$ is the energy of the $n$th Landau level in a constant magnetic field, and $f_{\mathrm{FD}}$ is the Fermi-Dirac distribution function. The basis vectors $\boldsymbol{e}_{i}^{(n)}$ are given in Eq. (A4) of the Appendix. Since the pair production is a dynamical process, it is more convenient to use the equal-time formula. We emphasize that the covariant DHW functions can be obtained from the equaltime ones by applying an additional Fourier transformation in $t$, i.e., $t \rightarrow p_{0}$, and conversely, the equal-time DHW functions can be derived from the covariant ones by integrating over $p_{0}$. Here, we give the equal-time DHW functions,

$$
\begin{aligned}
\boldsymbol{G}_{1}(\mathbf{p})= & \sum_{n=0} \frac{m}{E_{p_{z}}^{(n)}} C_{1}^{(n)}\left(p_{z}\right) \boldsymbol{e}_{1}^{(n)}\left(p_{T}\right), \\
\boldsymbol{G}_{2}(\mathbf{p})= & \sum_{n=0} C_{2}^{(n)}\left(p_{z}\right) \boldsymbol{e}_{1}^{(n)}\left(p_{T}\right), \\
\boldsymbol{G}_{3}(\mathbf{p})= & \frac{p_{z}}{E_{p_{z}}^{(0)}} C_{1}^{(0)}\left(p_{z}\right) \boldsymbol{e}_{1}^{(0)}\left(p_{T}\right)+\sum_{n>0} C_{1}^{(n)}\left(p_{z}\right) \frac{1}{E_{p_{z}}^{(n)}} \\
& \times\left[p_{z} \boldsymbol{e}_{2}^{(n)}\left(p_{T}\right)+\sqrt{2 n e B} \boldsymbol{e}_{3}^{(n)}\left(\mathbf{p}_{T}\right)\right], \\
\boldsymbol{G}_{4}(\mathbf{p})= & 0 .
\end{aligned}
$$

Here, $C_{1}^{(n)}\left(p_{z}\right) \equiv \int d p_{0} E_{p_{z}}^{(n)} V^{(n)}\left(p_{0}, p_{z}\right)$ and $C_{2}^{(n)}\left(p_{z}\right) \equiv$ $\int d p_{0}\left(p_{0}+\mu\right) V^{(n)}\left(p_{0}, p_{z}\right)$, respectively. The $p_{0}$-integrals can be performed, yielding the result

$$
\begin{aligned}
& C_{1}^{(n)}\left(p_{z}\right)=\frac{2-\delta_{n 0}}{(2 \pi)^{3}}\left[f_{\mathrm{FD}}\left(E_{p_{z}}^{(n)}-\mu\right)+f_{\mathrm{FD}}\left(E_{p_{z}}^{(n)}+\mu\right)-1\right], \\
& C_{2}^{(n)}\left(p_{z}\right)=\frac{2-\delta_{n 0}}{(2 \pi)^{3}}\left[f_{\mathrm{FD}}\left(E_{p_{z}}^{(n)}-\mu\right)-f_{\mathrm{FD}}\left(E_{p_{z}}^{(n)}+\mu\right)+1\right] .
\end{aligned}
$$

The Fermi-Dirac distributions are the number densities in coordinate space for fermions/antifermions. The prefactor $2-\delta_{n 0}$ is the spin degeneracy of the various Landau levels. Comparing with Eq. (15) without the electromagnetic field, Eq. (29) has more nonvanishing components and depends on the Landau levels $n$. We will show later that in a constant magnetic field different Landau levels evolve independently.

\section{B. Equations of motion}

In the presence of a constant magnetic field, the operator for the generalized spatial differentiation, cf. second equation in Eq. (8), becomes $\mathbf{D}_{\mathbf{x}}=e \mathbf{B} \times \boldsymbol{\nabla}_{\mathbf{p}}$, where the ordinary spatial gradient $\boldsymbol{\nabla}_{\mathbf{x}}$ has been dropped, since all considered fields are spatially homogeneous and the system is translation invariant.

The lowest Landau level is special since we only need the basis vector $\boldsymbol{e}_{1}^{(0)}\left(p_{T}\right)$ to describe the dynamics in the lowest Landau level. The reason is that, in a constant magnetic field, $\boldsymbol{e}_{1}^{(0)}\left(p_{T}\right)$ is an eigenvector for all operators $D_{t}, M_{1}, M_{2}$ appearing in the equation of motion (11),

$$
\begin{aligned}
& M_{1} \boldsymbol{e}_{1}^{(0)}\left(p_{T}\right)=2 p_{z} \boldsymbol{e}_{1}^{(0)}\left(p_{T}\right), \\
& M_{2} \boldsymbol{e}_{1}^{(0)}\left(p_{T}\right)=D_{t} \boldsymbol{e}_{1}^{(0)}\left(p_{T}\right)=0 .
\end{aligned}
$$

For the higher Landau levels, the situation is more complicated. In the last subsection, we have shown that the basis vectors $\boldsymbol{e}_{i}^{(n)}, i=1,2,3$, cf. Eq. (A4), are necessary to describe the equal-time DHW functions in a constant magnetic field. One can easily check that these basis vectors are not closed under the operator $M_{2}$ defined in 
Eq. (13). In order to construct a closed space under $M_{2}$, we need another basis vector, $\boldsymbol{e}_{4}^{(n)}$, the definition of which is also given in Eq. (A4). Acting $M_{1}, M_{2}$ onto these basis vectors and using the relations (A9) gives for all higher Landau levels $n>0$

$$
\begin{aligned}
& M_{1} \boldsymbol{e}_{i}^{(n)}\left(\mathbf{p}_{T}\right)=\sum_{j=1}^{4}\left(c_{1}^{(n)}\right)_{i j}^{T} \boldsymbol{e}_{j}^{(n)}\left(\mathbf{p}_{T}\right), \\
& M_{2} \boldsymbol{e}_{i}^{(n)}\left(\mathbf{p}_{T}\right)=\sum_{j=1}^{4}\left(c_{2}^{(n)}\right)_{i j}^{T} \boldsymbol{e}_{j}^{(n)}\left(\mathbf{p}_{T}\right),
\end{aligned}
$$

where the coefficient matrices are

$$
\begin{gathered}
c_{1}^{(n)} \equiv 2\left(\begin{array}{cccc}
0 & p_{z} & \sqrt{2 n e B} & 0 \\
p_{z} & 0 & 0 & 0 \\
\sqrt{2 n e B} & 0 & 0 & 0 \\
0 & 0 & 0 & 0
\end{array}\right), \\
c_{2}^{(n)} \equiv-2\left(\begin{array}{cccc}
0 & 0 & 0 & 0 \\
0 & 0 & 0 & \sqrt{2 n e B} \\
0 & 0 & 0 & -p_{z} \\
0 & -\sqrt{2 n e B} & p_{z} & 0
\end{array}\right) .
\end{gathered}
$$

Note that the transpose of these matrices appears in Eq. (32).

We have already seen in Eq. (29) that, when the electric field vanishes, the DHW functions can be expressed in terms of the basis vectors $\boldsymbol{e}_{i}^{(n)}$. Taking Eq. (29) as an initial condition, one can straightforwardly conclude that the DHW functions will stay in the space spanned by $\boldsymbol{e}_{i}^{(n)}$ when they evolve according to the equation of motion (11). This is because $D_{t}$ acting on $\boldsymbol{e}_{i}^{(n)}$ gives zero, while we have already seen that these basis vectors form a closed subset when acting with $M_{1,2}$ onto them; see Eq. (32). We thus make the ansatz

$\boldsymbol{G}_{i}(t, \mathbf{p})=f_{i}^{(0)}\left(t, p_{z}\right) \boldsymbol{e}_{1}^{(0)}\left(p_{T}\right)+\sum_{n>0} \sum_{j=1}^{4} f_{i j}^{(n)}\left(t, p_{z}\right) \boldsymbol{e}_{j}^{(n)}\left(\mathbf{p}_{T}\right)$,

where $i, j=1,2,3,4$. Since the magnetic field is assumed to be constant in time, the basis vectors $\boldsymbol{e}_{i}^{(n)}$ are also independent of time. Inserting Eq. (34) into the equation of motion (11) for the DHW functions, and using the orthogonality relations (A7) and (A8) for the basis vectors, we can derive the equations of motion for the functions $f_{i}^{(0)}$ and $f_{i j}^{(n)}$. For the lowest Landau level, we obtain
$D_{t}\left(\begin{array}{l}f_{1}^{(0)} \\ f_{2}^{(0)} \\ f_{3}^{(0)} \\ f_{4}^{(0)}\end{array}\right)\left(t, p_{z}\right)=2\left(\begin{array}{cccc}0 & 0 & 0 & p_{z} \\ 0 & 0 & 0 & 0 \\ 0 & 0 & 0 & -m \\ -p_{z} & 0 & m & 0\end{array}\right)\left(\begin{array}{c}f_{1}^{(0)} \\ f_{2}^{(0)} \\ f_{3}^{(0)} \\ f_{4}^{(0)}\end{array}\right)\left(t, p_{z}\right)$

The equations for the higher levels are

$$
\begin{aligned}
D_{t}\left(\begin{array}{l}
\boldsymbol{f}_{1}^{(n)} \\
\boldsymbol{f}_{2}^{(n)} \\
\boldsymbol{f}_{3}^{(n)} \\
\boldsymbol{f}_{4}^{(n)}
\end{array}\right)\left(t, p_{z}\right)= & \left(\begin{array}{cccc}
0 & 0 & 0 & c_{1}^{(n)} \\
0 & 0 & -c_{2}^{(n)} & 0 \\
0 & -c_{2}^{(n)} & 0 & -2 m \\
-c_{1}^{(n)} & 0 & 2 m & 0
\end{array}\right) \\
& \times\left(\begin{array}{l}
\boldsymbol{f}_{1}^{(n)} \\
\boldsymbol{f}_{2}^{(n)} \\
\boldsymbol{f}_{3}^{(n)} \\
\boldsymbol{f}_{4}^{(n)}
\end{array}\right)\left(t, p_{z}\right),
\end{aligned}
$$

where $\boldsymbol{f}_{i}^{(n)} \equiv\left(f_{i 1}^{(n)}, f_{i 2}^{(n)}, f_{i 3}^{(n)}, f_{i 4}^{(n)}\right)^{T}$ is a four-dimensional column vector. We observe that, on account of the orthogonality relations (A7) and (A8), the equations for the different Landau levels separate from each other, which greatly facilitates the solution of the equations of motion.

\section{Lowest Landau level}

The spin of the fermion in the lowest Landau level with positive/negative charge is parallel/antiparallel to the magnetic field. The equation for $f_{2}^{(0)}$, cf. the second line in Eq. (35), decouples from the other equations and gives rise to the conservation of net fermion number in the lowest Landau level. In order to see this, we note that the net fermion-number density $\mathcal{V}_{0}(t, \mathbf{p})$ is the first component of $\boldsymbol{G}_{2}$ in Eq. (12). The lowest Landau level contributes just $f_{2}^{(0)}\left(t, p_{z}\right) \Lambda_{+}^{(0)}\left(p_{T}\right)$, cf. Eqs. (34) and (A4). Acting $D_{t}=\partial_{t}+e E_{0} \partial_{p_{z}}$ on that and integrating over $\mathbf{p}$ yields with the definition $n^{(0)} \equiv \int d^{3} \mathbf{p} f_{2}^{(0)}\left(t, p_{z}\right) \Lambda_{+}^{(0)}\left(p_{T}\right)$, the conservation law

$$
\partial_{t} n^{(0)}=\int d^{3} \mathbf{p}\left[D_{t} f_{2}^{(0)}\left(t, p_{z}\right)\right] \Lambda_{+}^{(0)}\left(p_{T}\right)=0,
$$

where we have integrated by parts and neglected the boundary term. The equation $D_{t} f_{2}^{(0)}\left(t, p_{z}\right)=0$, together with $\left.f_{2}^{(0)}\left(t, p_{z}\right)\right|_{E_{0} \rightarrow 0}=C_{2}^{(n)}\left(p_{z}\right)$, has the special solution

$$
f_{2}^{(0)}\left(t, p_{z}\right)=C_{2}^{(0)}\left(p_{z}-e E_{0} t\right) .
$$

This solution describes an overall acceleration of all charged particles. We note that in this paper we focus on a free 
fermion gas, so there are no collisions to prevent the acceleration.

The equations of motion for the other three functions $f_{i=1,3,4}^{(0)}$ are coupled with each other. In order to simplify the problem, we make an ansatz which splits off the thermal distribution functions,

$$
\left\{f_{1}^{(0)}, f_{3}^{(0)}, f_{4}^{(0)}\right\}=\left\{\chi_{1}^{(0)}, \chi_{2}^{(0)}, \chi_{3}^{(0)}\right\} C_{1}^{(0)}\left(p_{z}-e E_{0} t\right),
$$

where $C_{1}^{(0)}$ is defined in Eq. (30). Here, $p_{z}+e A(t)=p_{z}-$ $e E_{0} t$ is the canonical momentum. When acting $D_{t}$ on $f_{i}^{(0)}$, we only need to consider its effect on $\chi_{i}$, because $D_{t} C_{1}^{(0)}\left(p_{z}-e E_{0} t\right)=0$. Thus, the equations of motion for the $\chi_{i}$ are the same as the one for the corresponding $f_{i}^{(0)}$, cf. Eq. (35),

$D_{t}\left(\begin{array}{l}\chi_{1}^{(0)} \\ \chi_{2}^{(0)} \\ \chi_{3}^{(0)}\end{array}\right)\left(t, p_{z}\right)=2\left(\begin{array}{ccc}0 & 0 & p_{z} \\ 0 & 0 & -m \\ -p_{z} & m & 0\end{array}\right)\left(\begin{array}{l}\chi_{1}^{(0)} \\ \chi_{2}^{(0)} \\ \chi_{3}^{(0)}\end{array}\right)\left(t, p_{z}\right)$.

Comparing the ansatz (39) with the initial condition (29), i.e., for $E_{0} \rightarrow 0$, we find

$$
\left.\left(\begin{array}{c}
\chi_{1}^{(0)} \\
\chi_{2}^{(0)} \\
\chi_{3}^{(0)}
\end{array}\right)\left(t, p_{z}\right)\right|_{E_{0} \rightarrow 0}=\frac{1}{E_{p_{z}}^{(0)}}\left(\begin{array}{c}
m \\
p_{z} \\
0
\end{array}\right)
$$

The system (40) of PDEs with the initial condition (41) coincides with the PDE system (24) in a pure electric field (substituting $\chi_{1} \rightarrow \chi_{2}^{(0)}, \chi_{2} \rightarrow \chi_{1}^{(0)}$, and $\chi_{3} \rightarrow-\chi_{3}^{(0)}$ and setting $\left.p_{T}^{2}=0\right)$. One can therefore immediately give the solution for a constant electric field $E(t)=E_{0}$,

$$
\left(\begin{array}{c}
\chi_{1}^{(0)} \\
\chi_{2}^{(0)} \\
\chi_{3}^{(0)}
\end{array}\right)\left(p_{z}\right)=\left(\begin{array}{c}
\frac{m}{\sqrt{2 e E_{0}}} d_{2}\left(\eta^{(0)}, \sqrt{\frac{2}{e E_{0}}} p_{z}\right) \\
d_{1}\left(\eta^{(0)}, \sqrt{\frac{2}{e E_{0}}} p_{z}\right) \\
-\frac{m}{\sqrt{2 e E_{0}}} d_{3}\left(\eta^{(0)}, \sqrt{\frac{2}{e E_{0}}} p_{z}\right)
\end{array}\right),
$$

with $d_{i}$ defined in Eq. (A1) and $\eta^{(0)}=m^{2} / e E_{0}$. Multiplying Eq. (42) with $\left.C_{1}^{(0)}\left(p_{z}-e E_{0} t\right)\right)$ gives the functions $f_{1}^{(0)}, f_{3}^{(0)}$, and $f_{4}^{(0)}$ in a constant electric field,

$$
\begin{aligned}
& \left(\begin{array}{c}
f_{1}^{(0)} \\
f_{3}^{(0)} \\
f_{4}^{(0)}
\end{array}\right)\left(p_{z}\right) \\
& =\left(\begin{array}{c}
\frac{m}{\sqrt{2 e E_{0}}} d_{2}\left(\eta^{(0)}, \sqrt{\frac{2}{e E_{0}}} p_{z}\right) C_{1}^{(0)}\left(p_{z}-e E_{0} t\right) \\
d_{1}\left(\eta^{(0)}, \sqrt{\frac{2}{e E_{0}}} p_{z}\right) C_{1}^{(0)}\left(p_{z}-e E_{0} t\right) \\
-\frac{m}{\sqrt{2 e E_{0}}} d_{3}\left(\eta^{(0)}, \sqrt{\frac{2}{e E_{0}}} p_{z}\right) C_{1}^{(0)}\left(p_{z}-e E_{0} t\right)
\end{array}\right) .
\end{aligned}
$$

Inserting these functions into Eq. (34), one obtains the contribution from the lowest Landau level to the DHW functions.

\section{Higher Landau levels}

For the higher Landau levels $(n>0)$, we can read off from Eqs. (29) and (34) that, when switching off the electric field, the only functions which do not vanish are $f_{11}^{(n)}, f_{21}^{(n)}$, $f_{32}^{(n)}$, and $f_{33}^{(n)}$. Writing down the equations of motion (36) for the $f_{i j}^{(n)}$ functions for the higher Landau levels using Eq. (33), we observe that $f_{24}^{(n)}, f_{42}^{(n)}$, and $f_{43}^{(n)}$ couple with $f_{11}^{(n)}, f_{32}^{(n)}$, and $f_{33}^{(n)}$ in the presence of an electric field. The corresponding six basis functions form a closed subspace. The other nine functions are decoupled to three independent groups, $\left\{f_{12}^{(n)}, f_{13}^{(n)}, f_{31}^{(n)}, f_{41}^{(n)}\right\},\left\{f_{22}^{(n)}, f_{23}^{(n)}, f_{34}^{(n)}, f_{44}^{(n)}\right\}$ and $\left\{f_{14}^{(n)}\right\}$, and each forms a closed set of homogeneous PDEs. However, since all of them have vanishing values when the electric field is zero, all of them will stay zero during the further evolution, even after switching on the electric field.

In the following, we therefore focus on the seven nontrivial functions $f_{11}^{(n)}, f_{21}^{(n)}, f_{24}^{(n)}, f_{32}^{(n)}, f_{33}^{(n)}, f_{42}^{(n)}$, and $f_{43}^{(n)}$. The equation for $f_{21}^{(n)}, D_{t} f_{21}^{(n)}=0$, decouples from the others. As discussed in the previous subsection, this equation is nothing but the conservation of net charge in each Landau level. The solution is

$$
f_{21}^{(n)}\left(t, p_{z}\right)=C_{2}^{(n)}\left(p_{z}-e E_{0} t\right),
$$

where $p_{z}-e E_{0} t$ describes the overall acceleration of all existing particles by the electric field in the $z$-direction.

As already mentioned above, the other six functions, $f_{11}^{(n)}, f_{24}^{(n)}, f_{32}^{(n)}, f_{33}^{(n)}, f_{42}^{(n)}$, and $f_{43}^{(n)}$, satisfy a six-dimensional system of PDEs. They can be further decoupled by introducing the following linear combinations, 


$$
\left(\begin{array}{cc}
g_{1}^{(n)} & g_{3}^{(n)} \\
g_{4}^{(n)} & g_{2}^{(n)}
\end{array}\right)=\frac{1}{m^{(n)}}\left(\begin{array}{cc}
m & \sqrt{2 n e B} \\
\sqrt{2 n e B} & -m
\end{array}\right)\left(\begin{array}{ll}
f_{11}^{(n)} & f_{24}^{(n)} \\
f_{33}^{(n)} & f_{42}^{(n)}
\end{array}\right),
$$

where the effective mass at level $n$ is $m^{(n)} \equiv \sqrt{m^{2}+2 n e B}$. Then, we get the following two groups of equations:

$$
\begin{aligned}
D_{t}\left(\begin{array}{c}
g_{1}^{(n)} \\
g_{2}^{(n)} \\
f_{32}^{(n)}
\end{array}\right)\left(t, p_{z}\right) \\
=2\left(\begin{array}{ccc}
0 & -p_{z} & 0 \\
p_{z} & 0 & -m^{(n)} \\
0 & m^{(n)} & 0
\end{array}\right)\left(\begin{array}{c}
g_{1}^{(n)} \\
g_{2}^{(n)} \\
f_{32}^{(n)}
\end{array}\right)\left(t, p_{z}\right),
\end{aligned}
$$

and

$D_{t}\left(\begin{array}{c}g_{3}^{(n)} \\ g_{4}^{(n)} \\ f_{43}^{(n)}\end{array}\right)\left(t, p_{z}\right)=2\left(\begin{array}{ccc}0 & -p_{z} & 0 \\ p_{z} & 0 & m^{(n)} \\ 0 & -m^{(n)} & 0\end{array}\right)\left(\begin{array}{c}g_{3}^{(n)} \\ g_{4}^{(n)} \\ f_{43}^{(n)}\end{array}\right)\left(t, p_{z}\right)$.

In this way, $g_{1}^{(n)}, g_{2}^{(n)}$, and $f_{32}^{(n)}$ decouple from $g_{3}^{(n)}, g_{4}^{(n)}$, and $f_{43}^{(n)}$. When the electric field vanishes, we find from Eqs. (29), (34), and (45) that $g_{3}^{(n)}, g_{4}^{(n)}$, and $f_{43}^{(n)}$ vanish. Under the time evolution determined by Eq. (47), this will remain the case after switching on $\mathbf{E}$. Therefore, we only need to focus on the equations for $g_{1}^{(n)}, g_{2}^{(n)}$, and $f_{32}^{(n)}$. Analogous to the treatment of the lowest Landau level, we assume that the solutions have the following form:

$\left\{g_{1}^{(n)}, g_{2}^{(n)}, f_{32}^{(n)}\right\}=\left\{\chi_{1}^{(n)}, \chi_{2}^{(n)}, \chi_{3}^{(n)}\right\} C_{1}^{(n)}\left(p_{z}-e E_{0} t\right)$.

Since $D_{t} C_{1}^{(n)}\left(p_{z}-e E_{0} t\right)=0$, the system of PDEs for $\left\{\chi_{1}^{(n)}, \chi_{2}^{(n)}, \chi_{3}^{(n)}\right\}$ reads

$D_{t}\left(\begin{array}{c}\chi_{1}^{(n)} \\ \chi_{2}^{(n)} \\ \chi_{3}^{(n)}\end{array}\right)\left(t, p_{z}\right)=2\left(\begin{array}{ccc}0 & -p_{z} & 0 \\ p_{z} & 0 & -m^{(n)} \\ 0 & m^{(n)} & 0\end{array}\right)\left(\begin{array}{l}\chi_{1}^{(n)} \\ \chi_{2}^{(n)} \\ \chi_{3}^{(n)}\end{array}\right)\left(t, p_{z}\right)$.

The initial values can be deduced by first reading off the functions $f_{i j}^{(n)}$ via a comparison of Eq. (29) and Eq. (34) and then using Eq. (45),

$$
\left.\left(\begin{array}{c}
\chi_{1}^{(n)} \\
\chi_{2}^{(n)} \\
\chi_{3}^{(n)}
\end{array}\right)\left(t, p_{z}\right)\right|_{E_{0} \rightarrow 0}=\frac{1}{E_{p_{z}}^{(n)}}\left(\begin{array}{c}
m^{(n)} \\
0 \\
p_{z}
\end{array}\right)
$$

The system (49) of PDEs and the initial condition (50) coincide with the PDE system (24) in a pure electric field (replacing $\chi_{1} \rightarrow \chi_{3}^{(n)}, \chi_{2} \rightarrow \chi_{1}^{(n)}, \chi_{3} \rightarrow \chi_{2}^{(n)}$, and setting $\left.p_{T}^{2}=2 n e B\right)$. Then, the solutions for a constant electric field $E(t)=E_{0}$ are straightforward to write down,

$$
\left(\begin{array}{c}
\chi_{1}^{(n)} \\
\chi_{2}^{(n)} \\
\chi_{3}^{(n)}
\end{array}\right)(\mathbf{p})=\left(\begin{array}{c}
\frac{m^{(n)}}{\sqrt{2 e E_{0}}} d_{2}\left(\eta^{(n)}, \sqrt{\frac{2}{e E_{0}}} p_{z}\right) \\
\frac{m^{(n)}}{\sqrt{2 e E_{0}}} d_{3}\left(\eta^{(n)}, \sqrt{\frac{2}{e E_{0}}} p_{z}\right) \\
d_{1}\left(\eta^{(n)}, \sqrt{\frac{2}{e E_{0}}} p_{z}\right)
\end{array}\right),
$$

with $d_{i}$ defined in Eq. (A1) and $\eta^{(n)}=\left(m^{2}+2 n e B\right) /\left(e E_{0}\right)$.

Now that we have found the solution for the $\chi_{i}^{(n)}$, we can insert it into the ansatz (48) and obtain $g_{1}^{(n)}, g_{2}^{(n)}$, and $f_{32}^{(n)}$. Then, using the inverse of the transformation (45), one can compute all nonvanishing functions,

$$
\begin{aligned}
\left(\begin{array}{c}
f_{11}^{(n)} \\
f_{33}^{(n)}
\end{array}\right)= & \left(\begin{array}{c}
m \\
\sqrt{2 n e B}
\end{array}\right) \frac{1}{\sqrt{2 e E_{0}}} d_{2}\left(\eta^{(n)}, \sqrt{\frac{2}{e E_{0}}} p_{z}\right) \\
& \times C_{1}^{(n)}\left(p_{z}-e E_{0} t\right), \\
\left(\begin{array}{c}
f_{24}^{(n)} \\
f_{42}^{(n)}
\end{array}\right)= & \left(\begin{array}{c}
\sqrt{2 n e B} \\
-m
\end{array}\right) \frac{1}{\sqrt{2 e E_{0}}} d_{3}\left(\eta^{(n)}, \sqrt{\frac{2}{e E_{0}}} p_{z}\right) \\
& \times C_{1}^{(n)}\left(p_{z}-e E_{0} t\right), \\
f_{32}^{(n)}= & d_{1}\left(\eta^{(n)}, \sqrt{\frac{2}{e E_{0}}} p_{z}\right) C_{1}^{(n)}\left(p_{z}-e E_{0} t\right),
\end{aligned}
$$

together with $f_{21}^{(n)}$ from Eq. (44). The remaining ten functions are zero.

\section{PAIR-PRODUCTION RATE}

In the last section, we have derived the DHW functions in constant electric and magnetic fields. In this section, we will relate the DHW functions to pair production. Note that, in the presence of an electric field, the system cannot remain in thermodynamical equilibrium.

Let us first consider a multipair system, where the particles are described by the plane-wave solutions of the free Dirac equation. Inserting these wave functions into the definition of the Wigner function and then projecting onto the unit 
matrix and the gamma matrices $\boldsymbol{\gamma}$, we obtain the contribution of fermion/antifermion pairs to the DHW functions [54],

$\mathcal{F}=\frac{2 m}{E_{\mathbf{p}}}\left[n_{\text {pair }}(\mathbf{p})-1\right], \quad \mathcal{V}=\frac{2 \mathbf{p}}{E_{\mathbf{p}}}\left[n_{\text {pair }}(\mathbf{p})-1\right]$,

where $n_{\text {pair }}(\mathbf{p})$ is the number density of pairs in phase space. The Pauli principle implies that $0 \leq n_{\text {pair }}(\mathbf{p}) \leq 1$. The density of pairs will change due to the pair-production process caused by the electric field. The corresponding rate is given by

$$
\frac{d}{d t} n_{\text {pair }}=\frac{1}{2} \frac{d}{d t} \int d^{3} \mathbf{p} \frac{m \mathcal{F}+\mathbf{p} \cdot \mathcal{V}}{E_{\mathbf{p}}}
$$

where $n_{\text {pair }}=\int d^{3} \mathbf{p} n_{\text {pair }}(\mathbf{p})$ is the number of pairs. Equation (54) can be proven by inserting Eq. (53) into the right-hand side.

Analogously, for a multipair system in a constant background magnetic field, the on-shell energy is $E_{p_{z}}^{(n)}=$ $\sqrt{m^{2}+p_{z}^{2}+2 n e B}$. If there is pair production by an electric field in the system, its rate in the $n$th Landau level can then be calculated via

$$
\frac{d}{d t} n_{\text {pair }}^{(n)}=\frac{1}{2} \frac{d}{d t} \int d^{3} \mathbf{p} \frac{m \mathcal{F}^{(n)}+\mathbf{p} \cdot \mathcal{V}^{(n)}}{E_{p_{z}}^{(n)}} .
$$

Here, $\mathcal{F}^{(n)}$ and $\mathcal{V}^{(n)}$ represent the DHW functions corresponding to the $n$th Landau level. Employing Eq. (12) and the ansatz (34), we get

$$
\begin{aligned}
\frac{d}{d t} n_{\text {pair }}^{(n)}= & \frac{1}{2} \frac{d}{d t} \int d^{3} \mathbf{p}\left[\frac{\eta^{(n)}}{E_{p_{z}}^{(n)}} \sqrt{\frac{e E_{0}}{2}} d_{2}\left(\eta^{(n)}, \sqrt{\frac{2}{e E_{0}}} p_{z}\right)\right. \\
& \left.+\frac{p_{z}}{E_{p_{z}}^{(n)}} d_{1}\left(\eta^{(n)}, \sqrt{\frac{2}{e E_{0}}} p_{z}\right)\right] \\
& \times C_{1}^{(n)}\left(p_{z}-e E_{0} t\right) \Lambda_{+}^{(n)}\left(p_{T}\right),
\end{aligned}
$$

where $C_{1}^{(n)}$ is given by Eq. (30). The integration over $\mathbf{p}_{T}$ can be performed using Eq. (A6). Replacing the kinetic momentum $p_{z}$ by the canonical momentum $q_{z}=p_{z}-e E_{0} t$, we obtain the pair-production rate in the $n$th Landau level in parallel electric and magnetic fields and a thermal background,

$$
\begin{aligned}
\frac{d}{d t} n_{\mathrm{pair}}^{(n)}= & \int d q_{z}\left[1-f_{\mathrm{FD}}\left(E_{q_{z}}^{(n)}-\mu\right)-f_{\mathrm{FD}}\left(E_{q_{z}}^{(n)}+\mu\right)\right] \\
& \times \frac{d}{d t} n_{\mathrm{vac}}^{(n)}\left(t, q_{z}\right),
\end{aligned}
$$

where $\frac{d}{d t} n_{\mathrm{vac}}^{(n)}\left(p_{z}\right)$ is the pair-production rate in vacuum for given quantum numbers $p_{z}$ and $n$,

$$
\begin{aligned}
& \frac{d}{d t} n_{\text {vac }}^{(n)}\left(t, q_{z}\right) \\
& =-\left(1-\frac{\delta_{n 0}}{2}\right) \frac{e^{2} B E_{0}}{(2 \pi)^{2}} \\
& \quad \times \frac{d}{d q_{z}}\left\{\frac{\eta^{(n)}}{E_{q_{z}+e E_{0} t}^{(n)}} \sqrt{\frac{e E_{0}}{2}} d_{2}\left[\eta^{(n)}, \sqrt{\frac{2}{e E_{0}}}\left(q_{z}+e E_{0} t\right)\right]\right. \\
& \left.\quad+\frac{q_{z}+e E_{0} t}{E_{q_{z}+e E_{0} t}^{(n)}} d_{1}\left[\eta^{(n)}, \sqrt{\frac{2}{e E_{0}}}\left(q_{z}+e E_{0} t\right)\right]\right\} .
\end{aligned}
$$

The Fermi-Dirac distributions in the square bracket in Eq. (57) describe the suppression of pair production due to the Pauli exclusion principle. Summing Eq. (57) over all Landau levels yields the total pair-production rate.

In a medium in which the chemical potential is zero but the temperature is nonzero, the suppression factor is $1-2 f_{\mathrm{FD}}\left(E_{q_{z}}^{(n)}\right)=\tanh \frac{\beta E_{q_{z}}^{(n)}}{2}$, which suppresses the production of pairs with small energies. This factor agrees with the result of Ref. [45]. However, the integral in Eq. (57) is hard to calculate numerically because the auxiliary functions $d_{1}$ and $d_{2}$ are highly oscillatory at large $q_{z}$, which makes the integration converge too slowly. This problem can be solved by separating the vacuum contribution from the thermal contribution. The pair-production rate in the $n$th Landau level in vacuum can be analytically calculated, using the asymptotic behavior of the $d_{1,2}$ functions, cf. Appendix,

$$
\begin{aligned}
\frac{d}{d t} n_{\text {vac }}^{(n)} & =\int d q_{z} \frac{d}{d t} n_{\text {vac }}^{(n)}\left(t, q_{z}\right) \\
& =-\left(1-\frac{\delta_{n 0}}{2}\right) \frac{e^{2} B E_{0}}{(2 \pi)^{2}}\left[d_{1}\left(\eta^{(n)},-\infty\right)+d_{1}\left(\eta^{(n)},+\infty\right)\right] \\
& =\left(1-\frac{\delta_{n 0}}{2}\right) \frac{e^{2} B E_{0}}{2 \pi^{2}} \exp \left(-\pi \frac{m^{2}+2 n e B}{e E_{0}}\right) .
\end{aligned}
$$

The total rate from all Landau levels is

$$
\frac{d}{d t} \sum_{n=0}^{\infty} n_{\text {vac }}^{(n)}=\frac{e^{2} E_{0} B}{4 \pi^{2}} \exp \left(-\frac{\pi m^{2}}{e E_{0}}\right) \operatorname{coth}\left(\frac{\pi B}{E_{0}}\right),
$$

which was previously derived in Refs. $[37,38,40]$. We see that the rate will be enhanced for $B \gg E_{0}$ compared to that without the magnetic field [8]. Similarly, we can also derive the production rate of chiral charge $d n_{5} / d t$ in a strong magnetic field, which gives the anomaly with the pair production and is consistent with the result of Ref. [58,59].

The thermal contribution in Eq. (57) for the $n$th Landau level is

$$
\begin{aligned}
\frac{d}{d t} n_{\text {thermal }}^{(n)}= & -\int d q_{z}\left[f_{\mathrm{FD}}\left(E_{q_{z}}^{(n)}-\mu\right)+f_{\mathrm{FD}}\left(E_{q_{z}}^{(n)}+\mu\right)\right] \\
& \times \frac{d}{d t} n_{\text {vac }}^{(n)}\left(t, q_{z}\right) .
\end{aligned}
$$



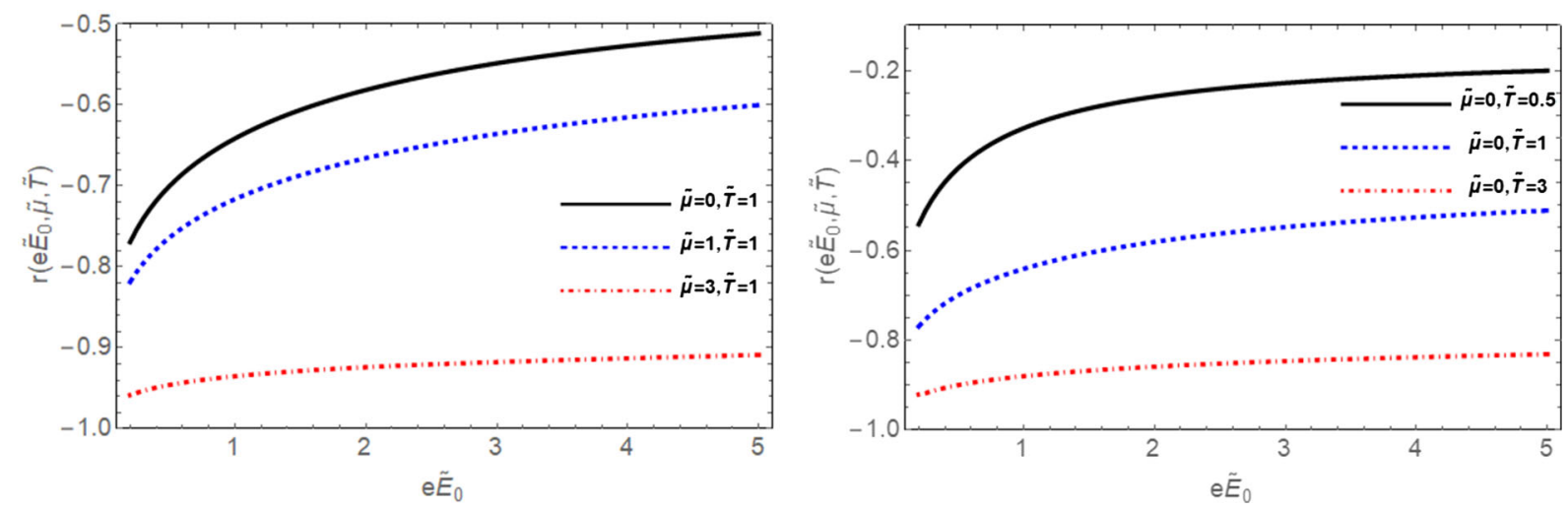

FIG. 1. The ratio of the thermal contribution to the total pair-production rate to the vacuum contribution for a constant electric field. Left panel: dependence on electric field strength for temperature $\tilde{T}=1$ and chemical potential $\tilde{\mu}=0,1,3$. Right panel: $\tilde{\mu}=0$ and $\tilde{T}=0.5,1,3$.

The Fermi-Dirac distributions provide an exponential suppression $\sim e^{-E_{q_{z}}^{(n)}}$ for large $q_{z}$; thus, the $q_{z}$-integral converges quickly. In order to show the thermal suppression in a physically intuitive way, we introduce the ratio $r$ of the thermal to the vacuum contribution. This ratio is a function of time $t$ and the three dimensionless parameters $e \tilde{E}_{0} \equiv \frac{e E_{0}}{\left[m^{(n)}\right]^{2}}, \tilde{T} \equiv \frac{T}{m^{(n)}}$, and $\tilde{\mu} \equiv \frac{\mu}{m^{(n)}}$, where $m^{(n)}=\sqrt{m^{2}+2 n e B}$ is the effective mass in the $n$th Landau level. The total pair-production rate in the $n$th Landau level is given by

$$
\frac{d}{d t} n_{\mathrm{pair}}^{(n)}=\left[1+r\left(t, \tilde{E}_{0}, \tilde{T}, \tilde{\mu}\right)\right] \frac{d}{d t} n_{\mathrm{vac}}^{(n)} .
$$

In order to show the thermal influence on pair production, we choose the time $t=0$, which is when the canonical momentum equals the kinetic one. Figure 1 shows the function $r\left(0, \tilde{E}_{0}, \tilde{T}, \tilde{\mu}\right)$ at finite dimensionless temperature and chemical potential. The values stay between -1 and 0 for all parameters considered, which describes the thermal suppression of pair production as demanded by the Pauli exclusion principle: a quantum state has a higher probability to be occupied at higher temperature or higher chemical potential; this occupation will block the production of new pairs with the same quantum numbers. When the electric field is strong enough, pairs with higher energies, which have smaller thermal occupation numbers, are more likely to be excited. Thus, the suppression is inversely proportional to the electric field strength.

\section{SUMMARY}

In this paper, we have analytically calculated the Wigner function as well as the Schwinger pair production in constant and parallel electric and magnetic fields. We have derived the equation of motion for the equal-time Wigner function, the 16 components of which, the so-called DHW functions, have definite physical meanings. One can relate the
Schwinger pair-production rate to some of these functions. For the case of a pure constant electric field, we took the vacuum values for the $16 \mathrm{DHW}$ functions as initial condition. Then, we obtained an analytic solution for the system of PDEs for the DHW functions. For parallel electric and magnetic fields, we adopted a similar method to calculate the DHW functions. We showed that the contributions of different Landau levels separate from each other. Under the replacement $p_{T}^{2} \rightarrow 2 n e B$, the system of PDEs and the condition when the electric field vanishes coincide with those in a pure electric field for each Landau level. This provides us with a new method for calculating the pair production in parallel electric and magnetic fields. Analytical solutions for the DHW functions for the case of constant electric and magnetic fields, together with the pair-production rate in each Landau level, are derived. Our results can be directly generalized to the case of finite temperature and chemical potential. The calculation shows that the pairproduction rate is thermally suppressed and the suppression is proportional to the thermodynamic variables $T$ and $\mu$. More energetic pairs can be created in a stronger electric field and are less likely to be Pauli blocked by the thermal distribution, and this leads to a decrease of the suppression factor.

The equation of motion for the Wigner function is equivalent to the Dirac equation if we adopt the classicalfield approximation. However, the Wigner function contains 16 independent components, which leads to a 16-dimensional system of PDEs. Due to advances in computer technology in the past few decades, it has become possible to numerically solve this PDE system in some simplified cases. In this paper, we have found a set of basis functions in the presence of a constant magnetic field. These basis functions provide us with a way to replace the continuous transverse momenta $p_{x}$ and $p_{y}$ by the discrete Landau level index $n$. The parameter space of the Wigner function is then simplified from six-dimensional phase space $(\mathbf{x}, \mathbf{p})$ to the four-dimensional space spanned by $\left(\mathbf{x}, p_{z}\right)$ plus one discrete parameter $n$, which makes the 

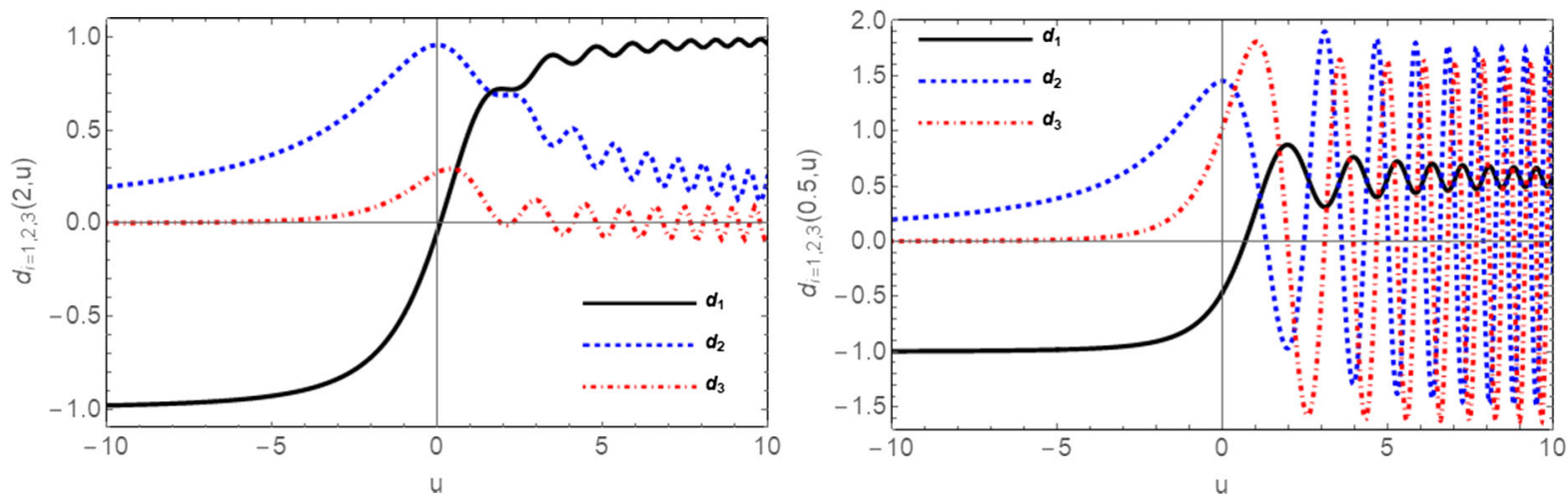

FIG. 2. $u$-dependence of the auxiliary functions $d_{i}(\eta, u), i=1,2,3$ for $\eta=2$ (left panel) and $\eta=0.5$ (right panel).

system of PDEs more amenable for a numerical solution. However, the case considered in this paper, i.e., homogeneous and parallel electromagnetic fields, is effectively only a $(1+1)$-dimensional problem, whereas the fields in real experiments are more likely to be space-time dependent. Nevertheless, the way to decompose the Wigner function presented here may inspire future works and may be a convenient starting point for the Wigner-function approach.

\section{ACKNOWLEDGMENTS}

Q.W. is supported in part by the 973 program under Grant No. 2015CB856902 and by NSFC under Grant No. 11535012. X. L. S. is supported in part by China Scholarship Council and the Deutsche Forschungsgemeinschaft through Grant no. CRC-TR 211 "Strong-interaction matter under extreme conditions." D. H. R. acknowledges support by the High-End Visiting Expert project of the State Administration of Foreign Experts Affairs of China and by the Deutsche Forschungsgemeinschaft, German Research Foundation) through the Collaborative Research Center TransRegio 211 "Strong-interaction matter under extreme conditions," Project No. 315477589-TRR 211.

\section{APPENDIX: AUXILIARY FUNCTIONS}

We have introduced three auxiliary functions in the solutions for the DHW functions,

$$
\begin{aligned}
& d_{1}(\eta, u)=-1+e^{-\frac{\pi \eta}{4} \eta}\left|D_{-1-i \eta / 2}\left(-u e^{i \frac{\pi}{4}}\right)\right|^{2}, \\
& d_{2}(\eta, u)=e^{-\frac{\pi \eta}{4}} e^{i \frac{\pi}{4}} D_{-1-i \eta / 2}\left(-u e^{i \frac{\pi}{4}}\right) D_{i \eta / 2}\left(-u e^{-i \frac{\pi}{4}}\right)+\text { c.c. } \\
& d_{3}(\eta, u)=e^{-\frac{\pi \eta}{4}} e^{-i \frac{\pi}{4}} D_{-1-i \eta / 2}\left(-u e^{i \frac{\pi}{4}}\right) D_{i \eta / 2}\left(-u e^{-i \frac{\pi}{4}}\right)+\text { c.c. },
\end{aligned}
$$

where $D_{\nu}$ is the parabolic cylinder function. These functions satisfy the following differential equations:

$$
\begin{aligned}
& \frac{d}{d u} d_{1}(\eta, u)=\eta d_{3}(\eta, u), \\
& \frac{d}{d u} d_{2}(\eta, u)=-u d_{3}(\eta, u), \\
& \frac{d}{d u} d_{3}(\eta, u)=-2 d_{1}(\eta, u)+u d_{2}(\eta, u) .
\end{aligned}
$$

We plot the $d_{i}$ as function of $u$ in Fig. 2. We observe that all these functions are convergent for $u \rightarrow-\infty$, but only $d_{1}$ is obviously convergent for $u \rightarrow+\infty$. The functions $d_{2}$ and $d_{3}$ are highly oscillatory in a finite region for large $u$, and thus $d_{2} / u$ and $d_{3} / u$ converge to zero when $u \rightarrow+\infty$. Moreover, we have

$\lim _{u \rightarrow-\infty} d_{1}(\eta, u)=-1, \quad \lim _{u \rightarrow+\infty} d_{1}(\eta, u)=1-2 e^{-\pi \eta}$.

Four groups of basis vectors are used in the expansion of the DHW functions in a constant magnetic field. They are functions of the Landau-level index $n$ and the transverse momentum $\mathbf{p}_{T}$, or its modulus $p_{T}=\sqrt{\mathbf{p}_{T} \cdot \mathbf{p}_{T}}$, respectively,

$$
\begin{aligned}
& \boldsymbol{e}_{1}^{(n)}\left(p_{T}\right)=\left(\begin{array}{c}
\Lambda_{+}^{(n)}\left(p_{T}\right) \\
\mathbf{0}_{T} \\
\Lambda_{-}^{(n)}\left(p_{T}\right)
\end{array}\right), \quad \boldsymbol{e}_{2}^{(n)}\left(p_{T}\right)=\left(\begin{array}{c}
\Lambda_{-}^{(n)}\left(p_{T}\right) \\
\mathbf{0}_{T} \\
\Lambda_{+}^{(n)}\left(p_{T}\right)
\end{array}\right), \\
& \boldsymbol{e}_{3}^{(n)}\left(\mathbf{p}_{T}\right)=\frac{\sqrt{2 n e B}}{p_{T}^{2}} \Lambda_{+}^{(n)}\left(p_{T}\right)\left(\begin{array}{c}
0 \\
\mathbf{p}_{T} \\
0
\end{array}\right), \\
& \boldsymbol{e}_{4}^{(n)}\left(\mathbf{p}_{T}\right)=\frac{\sqrt{2 n e B}}{p_{T}^{2}} \Lambda_{+}^{(n)}\left(p_{T}\right)\left(\begin{array}{c}
0 \\
-p_{y} \\
p_{x} \\
0
\end{array}\right) .
\end{aligned}
$$

Here, the $\Lambda_{ \pm}^{(n)}$ functions are defined as 


$$
\Lambda_{ \pm}^{(n)}\left(p_{T}\right) \equiv \begin{cases}(-1)^{n}\left[L_{n}\left(\frac{2 p_{T}^{2}}{e B}\right) \mp L_{n-1}\left(\frac{2 p_{T}^{2}}{e B}\right)\right] \exp \left(-\frac{p_{T}^{2}}{e B}\right), & n>0 \\ 2 \exp \left(-\frac{p_{T}^{2}}{e B}\right), & n=0\end{cases}
$$

where $L_{n}(x)$ is the $n$th Laguerre polynomial. For the lowest Landau level, $n=0$, we have $\boldsymbol{e}_{3}^{(0)}=\boldsymbol{e}_{4}^{(0)}=0$ and $\boldsymbol{e}_{1}^{(0)}=\boldsymbol{e}_{2}^{(0)}$. These basis vectors allow us to separate the $\mathbf{p}_{T}$ dependence. When integrating over transverse momentum $\mathbf{p}_{T}, \Lambda_{+}^{(n)}\left(p_{T}\right)$ gives the density of states for Landau level $n$, while $\Lambda_{-}^{(n)}\left(p_{T}\right)$ gives zero for all $n>0$,

$$
\begin{aligned}
& \frac{1}{(2 \pi)^{2}} \int d^{2} \mathbf{p}_{T} \Lambda_{+}^{(n)}\left(p_{T}\right)=\frac{e B}{2 \pi}, \\
& \frac{1}{(2 \pi)^{2}} \int d^{2} \mathbf{p}_{T} \Lambda_{-}^{(n)}\left(p_{T}\right)=0, \quad(n \neq 0) .
\end{aligned}
$$

The basis vectors $\boldsymbol{e}_{i}^{(n)}$ for $i=1,2,3,4$ and $n=0,1,2, \ldots$ are orthogonal with respect to an inner product,

$$
\int d^{2} \mathbf{p}_{T} \boldsymbol{e}_{i}^{(m) T}\left(\mathbf{p}_{T}\right) \boldsymbol{e}_{j}^{(n)}\left(\mathbf{p}_{T}\right)=2 \pi e B \delta_{m n} \delta_{i j}
$$

for $n>0$, together with

$$
\begin{aligned}
& \int d^{2} \mathbf{p}_{T} \boldsymbol{e}_{1}^{(0) T}\left(p_{T}\right) \boldsymbol{e}_{1}^{(0)}\left(p_{T}\right)=4 \pi e B \\
& \int d^{2} \mathbf{p}_{T} \boldsymbol{e}_{i}^{(n) T}\left(\mathbf{p}_{T}\right) \boldsymbol{e}_{1}^{(0)}\left(p_{T}\right)=0
\end{aligned}
$$

We can also check that the functions $\Lambda_{ \pm}^{(n)}$ in Eq. (A5) satisfy the following relations,

$$
\begin{aligned}
& e B \partial_{p_{x}} \Lambda_{+}^{(n)}\left(p_{T}\right)=-2 p_{x} \Lambda_{-}^{(n)}\left(p_{T}\right) \\
& e B \partial_{p_{x}} \Lambda_{-}^{(n)}\left(p_{T}\right)=-2 p_{x}\left(1-\frac{2 n e B}{p_{T}^{2}}\right) \Lambda_{+}^{(n)}\left(p_{T}\right)
\end{aligned}
$$

which are used to derive Eqs. (31) and (32).
[1] W. Greiner, B. Muller, and J. Rafelski, Quantum Electrodynamics of Strong Fields (Springer Science+Business Media, New York, 1985).

[2] D. E. Kharzeev, L. D. McLerran, and H. J. Warringa, Nucl. Phys. A803, 227 (2008).

[3] J. Rafelski and B. Müller, Phys. Rev. Lett. 36, 517 (1976).

[4] K. Tuchin, Phys. Rev. C 91, 064902 (2015).

[5] H. Li, X.-1. Sheng, and Q. Wang, Phys. Rev. C 94, 044903 (2016).

[6] F. Sauter, Z. Phys. 69, 742 (1931).

[7] W. Heisenberg and H. Euler, Z. Phys. 98, 714 (1936).

[8] J. S. Schwinger, Phys. Rev. 82, 664 (1951).

[9] A. Ringwald, Phys. Lett. B 510, 107 (2001).

[10] A. Ringwald, arXiv:hep-ph/0112254.

[11] G. V. Dunne, Eur. Phys. J. D 55, 327 (2009).

[12] S. A. Smolyansky, G. Ropke, S. M. Schmidt, D. Blaschke, V. D. Toneev, and A. V. Prozorkevich, arXiv: hep-ph/9712377.

[13] S. M. Schmidt, D. Blaschke, G. Ropke, S. A. Smolyansky, A. V. Prozorkevich, and V. D. Toneev, Int. J. Mod. Phys. E 07, 709 (1998).

[14] Y. Kluger, E. Mottola, and J. M. Eisenberg, Phys. Rev. D 58, 125015 (1998).

[15] E. Brezin and C. Itzykson, Phys. Rev. D 2, 1191 (1970).
[16] V. S. Popov, Zh. Eksp. Teor. Fiz. 61, 1334 (1971) [Sov. Phys. JETP 34, 709 (1972)].

[17] V. S. Popov and M. S. Marinov, Yad. Fiz. 16, 809 (1972) [Sov. J. Nucl. Phys. 16, 449 (1973)].

[18] I. K. Affleck, O. Alvarez, and N. S. Manton, Nucl. Phys. B197, 509 (1982).

[19] S. P. Kim and D. N. Page, Phys. Rev. D 65, 105002 (2002).

[20] G. V. Dunne and Q.-h. Wang, Phys. Rev. D 74, 065015 (2006).

[21] F. Hebenstreit, R. Alkofer, and H. Gies, Phys. Rev. D 82, 105026 (2010).

[22] C. Kohlfuerst and R. Alkofer, Phys. Lett. B 756, 371 (2016).

[23] C. Kohlfuerst, Eur. Phys. J. Plus 133, 191 (2018).

[24] H. Gies and K. Klingmuller, J. Phys. A 39, 6415 (2006).

[25] C. Schneider, G. Torgrimsson, and R. Schuetzhold, Phys. Rev. D 98, 085009 (2018).

[26] J. Ambjorn and Y. Makeenko, Phys. Rev. D 85, 061901 (2012).

[27] Y. Sato and K. Yoshida, J. High Energy Phys. 04 (2013) 111.

[28] Y. Sato and K. Yoshida, J. High Energy Phys. 09 (2013) 134.

[29] G. V. Dunne and C. Schubert, Phys. Rev. D 72, 105004 (2005). 
[30] W.-T. Deng and X.-G. Huang, Phys. Rev. C 85, 044907 (2012).

[31] L. McLerran and V. Skokov, Nucl. Phys. A929, 184 (2014).

[32] A. Blinne and H. Gies, Phys. Rev. D 89, 085001 (2014).

[33] D. Berényi, S. Varró, V. V. Skokov, and P. Lévai, Phys. Lett. B 749, 210 (2015).

[34] T. Tajima and G. Mourou, Phys. Rev. ST Accel. Beams 5, 031301 (2002).

[35] N. B. Narozhny, S. S. Bulanov, V. D. Mur, and V. S. Popov, Phys. Lett. A 330, 1 (2004).

[36] R. A. Abramchuk and M. A. Zubkov, Phys. Rev. D 94, 116012 (2016).

[37] A. I. Nikishov, Zh. Eksp. Teor. Fiz. 57, 1210 (1969) [Sov. Phys. JETP 30, 660 (1970)].

[38] F. V. Bunkin and A. E. Kazakov, Dokl. Akad. Nauk Ser. Fiz. 193, 1274 (1970) [Sov. Phys. Dokl. 14, 678 (1970)].

[39] J. K. Daugherty and I. Lerche, Phys. Rev. D 14, 340 (1976).

[40] G. V. Dunne, Heisenberg-Euler effective lagrangians: Basics and extensions From Fields to Strings: Circumnavigating Theoretical Physics, edited by M. Shifman (World Scientific Publishing, Singapore, 2005), DOI: 10.1142/ 9789812775344_0014.

[41] J. X. Lu, J. High Energy Phys. 12 (2017) 076.

[42] J. X. Lu, Phys. Lett. B 788, 480 (2019).

[43] Q. Jia and J. X. Lu, Phys. Lett. B 789, 568 (2019).

[44] H. Gies, Phys. Rev. D 61, 085021 (2000).
[45] S. P. Kim, H. K. Lee, and Y. Yoon, Phys. Rev. D 79, 045024 (2009).

[46] O. Gould and A. Rajantie, Phys. Rev. D 96, 076002 (2017).

[47] W. Cai, K.-1. Li, and S.-w. Li, arXiv:1612.07087.

[48] E.-d. Guo and S. Lin, Phys. Rev. D 93, 105001 (2016).

[49] G. Cao and X.-G. Huang, Phys. Lett. B 757, 1 (2016).

[50] R.-h. Fang, J.-y. Pang, Q. Wang, and X.-n. Wang, Phys. Rev. D 95, 014032 (2017)

[51] S. Lin and L. Yang, Phys. Rev. D 98, 114022 (2018).

[52] D. Vasak, M. Gyulassy, and H. T. Elze, Ann. Phys. (N.Y.) 173, 462 (1987).

[53] H.-T. Elze and U. W. Heinz, Phys. Rep. 183, 81 (1989).

[54] I. Bialynicki-Birula, P. Gornicki, and J. Rafelski, Phys. Rev. D 44, 1825 (1991).

[55] P.-f. Zhuang and U. W. Heinz, Phys. Rev. D 57, 6525 (1998).

[56] E. V. Gorbar, V. A. Miransky, I. A. Shovkovy, and P. O. Sukhachov, J. High Energy Phys. 08 (2017) 103.

[57] X.-1. Sheng, D. H. Rischke, D. Vasak, and Q. Wang, Eur. Phys. J. A 54, 21 (2018).

[58] K. Fukushima, D. E. Kharzeev, and H. J. Warringa, Phys. Rev. Lett. 104, 212001 (2010).

[59] P. Copinger, K. Fukushima, and S. Pu, Phys. Rev. Lett. 121, 261602 (2018). 